OPEN ACCESS

Edited by:

Raul Vicente,

University of Tartu, Estonia

Reviewed by:

Jose De Jesus Rubio,

Instituto Politécnico

Nacional, Mexico

Jonathan M. Aitken,

University of Sheffield, United

Kingdom

*Correspondence:

Leonardo Trujillo

leonardo.trujillo@tectijuana.edu.mx;

Yazmin Maldonado

yaz.maldonado@tectijuana.edu.mx

Specialty section:

This article was submitted to Computational Intelligence,

a section of the journal

Frontiers in Robotics and Al

Received: 09 January 2018

Accepted: 12 March 2018

Published: 28 March 2018

Citation:

Torres N, Trujillo $L$ and Maldonado $Y$ (2018) Modeling Uncertainty for the

Double Standard Model Using a

Fuzzy Inference System.

Front. Robot. Al 5:31.

doi: 10.3389/frobt.2018.00031

\section{Modeling Uncertainty for the Double Standard Model Using a Fuzzy Inference System}

\author{
Noelia Torres, Leonardo Trujillo* and Yazmin Maldonado* \\ Departamento de Ingenieria Electrica y Electronica, Instituto Tecnologico de Tijuana, Tijuana, Mexico
}

This paper studies the issue of uncertainty in the ambulance location problem to cover the maximum number of demand points in a city. The work is based on the double standard model (DSM), a popular coverage model where two radii are considered to cover a percentage of the demand points twice. Uncertainty is introduced in the expected travel time between an ambulance and a demand point, before computing the optimal placement of ambulances in potential bases by solving the linear program posed by the DSM. The following three approaches are considered: (1) solving the DSM without uncertainty; (2) uncertainty in the travel time is based on triangular fuzzy set; and (3) a fuzzy inference system (FIS) with a rule base derived from the problem properties, which is the main contribution of this work. Results show that considering uncertainty can have a significant effect on the solutions for the DSM, with the solutions produced with the FIS approach achieving a higher total coverage of the demand. In conclusion, the proposed strategy could provide a reliable and effective tool to support decision making in the ambulance location problem by considering uncertainty in the ambulance travel times.

\footnotetext{
Keywords: ambulances, emergency medical services, bases, double standard model, triangular fuzzy set, fuzzy inference system
}

\section{INTRODUCTION}

In recent years, the development of computational support systems for emergency medical services (EMS) has attracted a growing amount of attention from researchers. In EMSs, a crucial factor that must be considered is response time. For example, let us consider cardiac arrest, the American Heart Association concluded that in the first 4-6 min after a person suffers a cardiac arrest she (he) can begin to suffer from permanent brain damage or even brain death. However, normal heart rhythm can be restored if advanced life support (ALS) is provided early. Studies have shown that the probability of a patient surviving is reduced by $7-10 \%$ with each passing minute in which defibrillation and ALS are not provided, and resuscitation is mostly unsuccessful after $10 \mathrm{~min}$ (EMSWorld.com, 2014).

Another example is traffic accident, where the number of deaths was estimated to be 1.25 million in 2013. Half of all fatalities caused by traffic accidents are pedestrians, cyclists, and motorcyclists. Death rates from such accidents are considerably higher in developing countries (OMG, 2015). Therefore, appropriate and timely response times to such incidents are indispensable in highly urban areas.

In 2013, the Red Cross of Tijuana (RCT) covered about 98\% of the EMS requests (Cruz Roja de Tijuana, 2012), providing medical attention to 37,000 emergency calls. It does this with 13 
ambulances that are distributed in 8 bases that cover a population of approximately 1.6 million people in an area of $1,243 \mathrm{~km}^{2}$ (INEGI, 2010). This means that, on average, each ambulance serves about 123,000 people and each base must cover an average of $155 \mathrm{~km}^{2}$. This contrasts, for instance, with the US, where by the 1990s there was about 1 ambulance per 51,000 inhabitants (Braun et al., 1990).

This work is motivated by the lack of resources and their sub-optimal use, which is evident when we consider that the average response time of RCT ambulances was approximately $14 \mathrm{~min}$ with an SD of $7 \mathrm{~min}$. Such performance is unsatisfactory, and there is a pressing need to optimize the use of all available resources.

In this work, the main objective is to study the ambulance location problem that seeks to determine where to place the available ambulances within a city. To do so, we use a well-known coverage model and solve it with linear programming. However, our contribution is to consider uncertainty in the estimated travel time between an ambulance and a possible demand point. In particular, we explore the use of fuzzy sets and a fuzzy inference system (FIS), which provide a natural way to describe uncertainty in a human readable form. The results show that the coverage provided by the solutions found can be substantially different, when uncertainty is explicitly considered.

This paper is organized as follows. Section "Background" presents a review of the state of the art. Section "Double Standard Model" describes the double standard model (DSM), which is the coverage model used in this work. Section "Travel Time Estimation for Ambulances" presents how uncertainty in the travel time is modeled in our work, with our main contribution being the use of an FIS. Section "Experiments and Results" presents the description of the experiments and obtained results. Finally, Section "Conclusion and Future Work" outlines the conclusion and describes future work.

\section{BACKGROUND}

Traditionally, the ambulance location problem deals with two types of decisions: (a) which sites in a city should be used as bases, and (b) determining how many ambulances should be placed in each base. What follows will deal mostly with models for this problem, while solution methods will not be covered in depth. Suffice it to say that many solution methods have been applied, including linear programming (MatLab, 2015) (which is also used in this work), Tabu search (Gendrau et al., 1997; Luke, 2014), evolutionary algorithms (Jones, 2002; Eiben and Smith, 2015), and Fuzzy logic (Zadeh, 2015).

Models for this problem can be divided into three main groups (Li et al., 2011): (1) covering models that focus on locating ambulances such that the demand can be covered within a certain amount of time; (2) p-median models that focus on minimizing the total (or average) distance between ambulance and demand points; and (3) p-center models that minimize the maximum distance between ambulances and demand points. Among the three groups, the coverage models are the most frequent and therefore reviewed next. These models are concerned with maximizing demand coverage, considering that a demand point is covered if it can be reached within a predefined time or distance by an ambulance, referred to as a coverage radius.

According to the literature, the first coverage model was the location set coverage problem (Toregas et al., 1971). It considers mandatory coverage, the goal is to determine the minimum amount of resources required so that all demand points are covered. Another well-known model is the maximum coverage location problem (Church and ReVelle, 1974), where given a limited number of resources the goal is to maximize the demand covered. Both of these models produce solutions where a demand point can be left uncovered once an ambulance response to a service. In the literature, there are two general proposals to overcome this drawback. The first is to provide multiple coverage, such as the DSM (Gendrau et al., 1997), the goal of which is to achieve full coverage within a large radius and maximize double coverage within a shorter radius. A second approach is to model the problem probabilistically, as in the maximum expected cover location problem and the maximum availability location problem.

The DSM considers a static number of ambulances, while a dynamic version has also been developed (Laporte et al., 2009). The multi-capacities ambulance location model was proposed in Shiah et al. (2009), which concurrently considers three different types of capacities: travel distance, populations, and the location EMS calls.

In the study by Schmid (2012), it was shown that solving the static location problem using fixed travel times may not suffice, due to the speed variations of the ambulances and because coverage areas change throughout the day. The problem was solved by the authors using a variable neighborhood search.

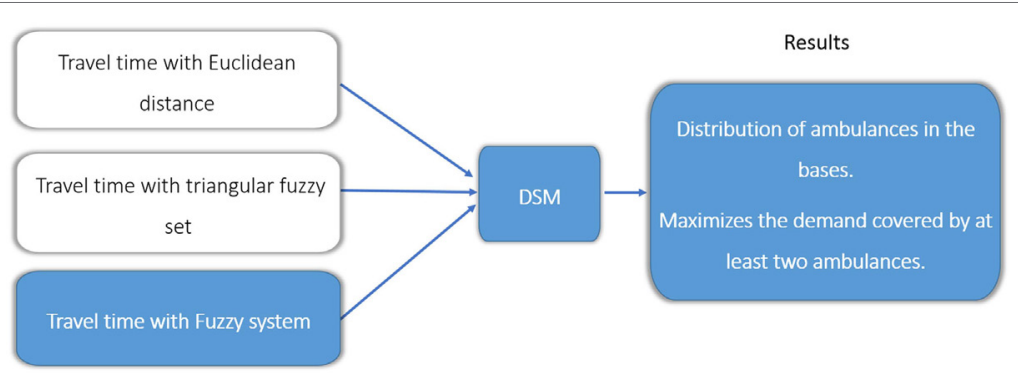

FIGURE 1 | Different methods used to estimate the ambulance travel times for the double standard model (DSM). 
Fuzzy logic as a solution method was used by Davari et al. (2011), who proposed the fuzzy maximum coverage location problem, which is solved by simulated annealing. Another fuzzy covering model is studied in the study by Rezaei and Zarandi (2011). The reduction of ambulance response time is studied using a hybrid approach in the study by Zarkeshzadeh et al. (2016). The method considers the rate of incoming emergency calls, available resources, the probability of hospitalization of patients, as well as the distances and locations of the emergency units.

As previous work on this topic, in 2017 we have reported on modeling the demand for EMS in Tijuana, Baja California, Mexico, followed by the optimization of the location of ambulances for the RCT. We used data from more than 10,000 emergency calls from 2013 to model and classify the demand for EMS in different scenarios that provide different perspectives of the demand throughout the city, considering factors such as the time of day or whether the incident occurred in a work day or an off-day (Dibene et al., 2017). In that work, the DSM was extended to generate robust solutions that could generalize to different scenarios. A total of 1,000 possible bases are considered and the solution was generated with integer linear programming. Results showed how the solutions found could improve coverage relative to the approach taken by the RCT at the time without requiring additional resources.

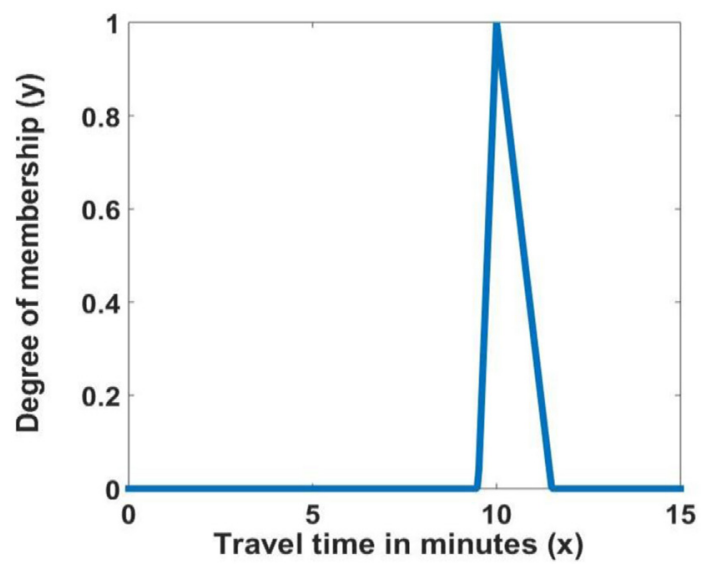

FIGURE 2 | Travel time membership function.

\section{DOUBLE STANDARD MODEL}

In the DSM, the objective function computes the demand covered twice within small radius $\left(r_{1}\right)$ time units. Below, we describe DSM problem and parameters, defined as follows.

Maximize:

$$
f(x)=\sum_{i \in l} d_{i} x_{i 2}
$$

Subject to:

$$
\begin{gathered}
\sum_{j \in J} \delta_{i j} y_{j} \geq 1 \forall i \in I \\
\sum_{i \in I} d_{i} x_{i 1} \alpha \sum_{i \in I} d_{i} \\
\sum_{j \in I} \gamma_{i j} y_{j} \geq x_{i 1}+x_{i 2} \quad \forall i \in I \\
x_{i 2} \leq x_{i 1} \quad \forall i \in I \\
\sum_{j \in J} \gamma_{i j}=p \\
y_{j} \leq p_{j} \quad \forall j \in J \\
\sum_{i \in I} \delta_{i j} z_{i j} \leq w_{j} y_{i j} \forall j \in J \\
\sum_{j \in J} \delta_{i j} z_{i j} \leq d_{i} \quad \forall i \in I \\
y_{j} \geq 0 \forall j \in J \\
z_{i j} \geq 0 \forall i \in I, \forall j \in J \\
x_{i, k} \in\{0,1\} \forall i \in I, k \in\{1,2\} \\
y_{j} \in \mathbb{Z} \forall j \in J \\
Z_{i j} \in \mathbb{Z} \forall i \in I, \forall j \in J
\end{gathered}
$$

where $i \in I$ : demand point; $j \in J$ : potential ambulance location; $r_{1}$ : small radius; $r_{2}$ : large radius; $p$ : total the available ambulances; $t_{i j}$ : the distance between the point of demand and the base, this variable is the travel time and is calculated using three models, described in the following section; $\alpha$ : percentage of total demand which must be covered by an ambulance located within $r_{1}$ radius; $d_{i}$ : total demand at patient location $i ; p_{j}$ : the largest

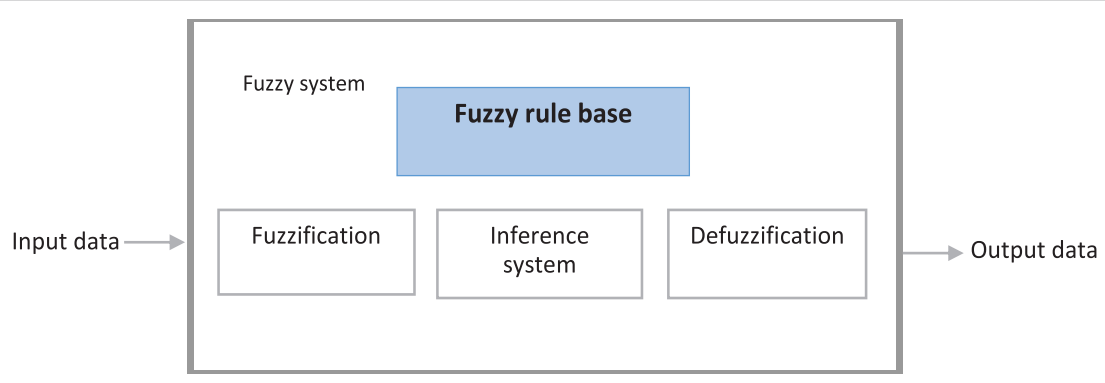

FIGURE 3 | Fuzzy inference system. 
number of ambulances at the ambulance location; $w_{j}$ : the capacity of each ambulance when it is placed in ambulance location $j$; $\delta_{i, j}=\left\{\begin{array}{cc}1 & t_{i j} \leq r_{2} \\ 0 & o . w\end{array}\right.$, if the distance between the point of demand and the base is less or equal to $r_{2}$, all demands are covered within the large radius; $\gamma_{i, j}=\left\{\begin{array}{cc}1 & t_{i j} \leq r_{2} \\ 0 & o . w\end{array}\right.$, if the distance between the demand point and the base is less than or equal to $r_{1}$, all demands are covered within the small radius; Variables: $y_{j}=$ the number of ambulance located at the vehicle location $j . x_{i 1}=1$, if the place $i$ is covered by one ambulance through the small radius. $x_{i 2}=11$,

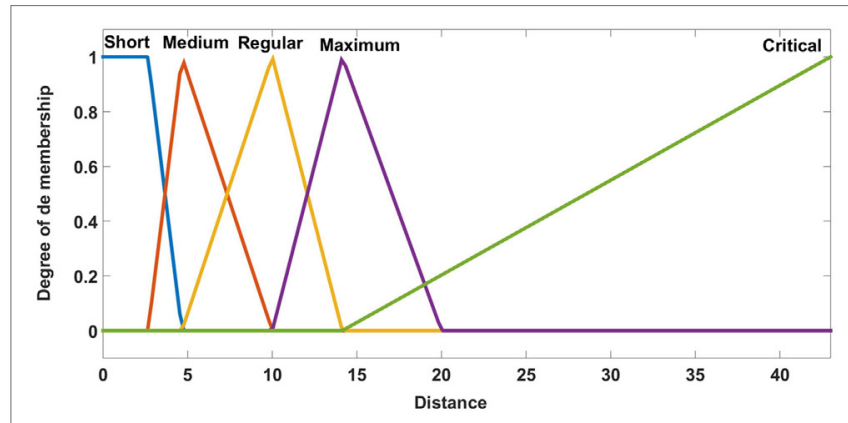

FIGURE 4 | Membership functions of distance.

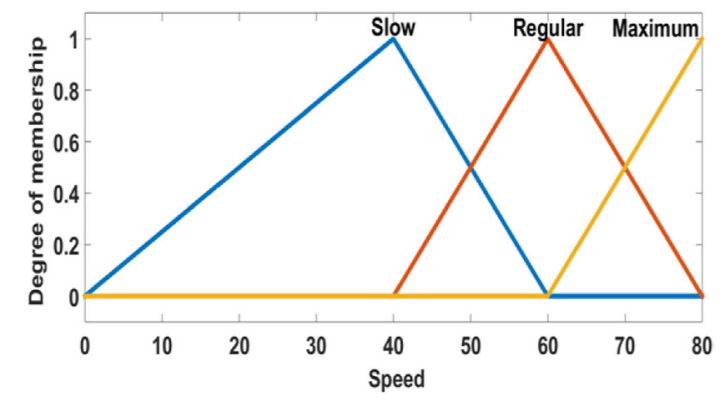

FIGURE 5 | Membership functions of speed. if the place $i$ is covered by two or more ambulance through the small radius. $z_{i j}=$ current demand at the patient location $i$ which is covered by one ambulance at the ambulance location $j$.

The goal of the objective function (1) is to maximize the demand covered by at least two ambulances within the small radius. Constraint (2) ensures that one ambulance covers each demand point within the larger radius $r_{2}$. Constraint (3) states that a fraction $\alpha$ of the total demand must be covered within the small radius. The left-hand side of constraint (4) has the number of ambulances covering demand point $i$ within $r_{2}$, and the right-hand side is equal to 1 if the demand point $i$ is covered exactly once within the small radius and equal to 2 if it is covered at least twice. Constraint (5) states that a demand point $i$ cannot be covered twice if it is not covered once. Constraints (4) and (5) state that if two ambulances are within $r_{1}$, the demand point is said to be covered twice. Constraint (6) states that all ambulances must be assigned to a base. Constraint (7) verifies the maximum number of ambulances at each base $j$. Constraint (8) ensures that the demand covered by base $j$ depends on the ambulance locations. Constraint (9) states that the total demand in demand point $i$ must be covered within the large radius $r_{2}$. Constraints (10) and (11) express that some variables cannot be negative and constraint (12) states that the coverage index is a binary variable. Constraint (13) shows that the total available ambulances is an integer. Finally, constraint (14) states that $z_{i j}$ must be an integer.

\section{TRAVEL TIME ESTIMATION FOR AMBULANCES}

The EMS providers prefer to assign the closest, in terms of travel time, available ambulance to respond to a new emergency. Thus, it is vital to have accurate estimates of the travel time of each ambulance to the emergency location. Therefore, travel times play a central role in positioning the ambulance bases (Laporte et al., 2009). The intuition behind this work is that if the variability in travel times is accounted for explicitly then EMS management and planning can be significantly improved. To show this, we use three different methods to calculate the ambulance travel time: (1) using fixed travel times (Laporte et al., 2009); (2) introducing

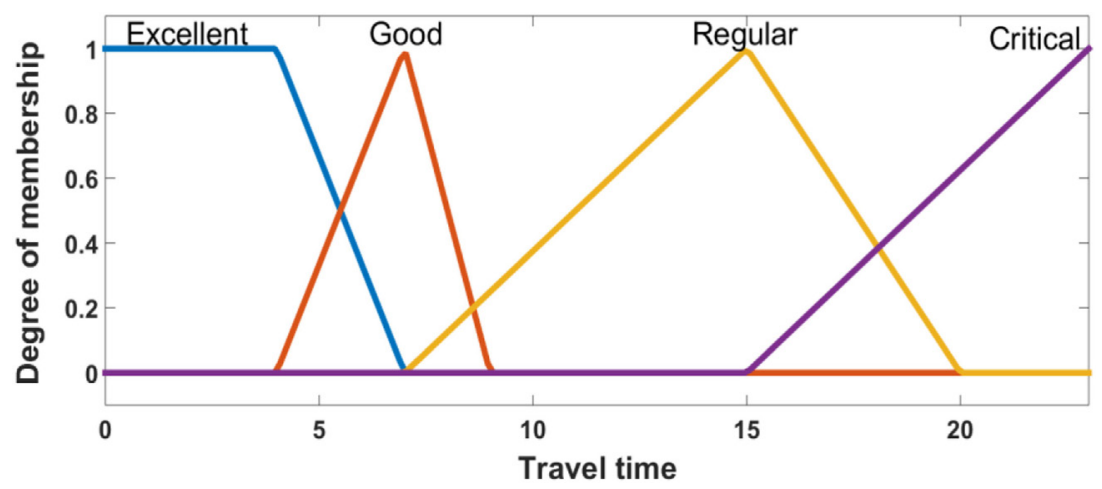

FIGURE 6 | Membership functions of travel time. 
uncertainty in the travel time using a triangular fuzzy set (TFS) as proposed by Lahijanian et al. (2016); and the main proposal in this work, (3) using an FIS to account for uncertainty in the travel time between an ambulance and a demand point. A simulation is setup to consider travel times in a road network, following the study by Lahijanian et al. (2016). Random data are generated in a hypothetical area of $900 \mathrm{~km}^{2}$, using 30 bases and 100 random demand points. Figure 1 shows the three versions of the DSM.

\section{Basic DSM Approach}

To calculate the travel time between a demand point and an ambulance, we used the Euclidian distance divided by speed, using three different speeds $(40,60$, and $80 \mathrm{~km} / \mathrm{h}$ ) for the ambulance.

\section{DSM With TFS}

The DSM with a TFS considers the uncertainty for the travel time between locations of the patients and the locations of the ambulances, based on the study by Lahijanian et al. (2016).

1. The first step is to convert the time to a triangular fuzzy number, adding uncertainty in the trip.

2. The TFS, shown in Figure 2, is defined as a function of $x$, where $y$ depends on three scalar parameters $a, b$, and $c$, defined as:

$$
\mu(x)=\left\{\begin{array}{ccc}
\frac{x-a}{b-a} & \text { if } & a \leq x \leq b \\
\frac{x-c}{b-c} & \text { if } & b \leq x \leq c \\
0 & & \text { Otherwise }
\end{array}\right.
$$

3. The defuzzification is done using the centroid method to obtain a crisp number, this is done by extracting the average from the triangular fuzzy number.

TABLE 1 | Matrix of inference rules used with the proposed fuzzy inference system.

\begin{tabular}{lllll}
\hline \multicolumn{5}{c}{ Input speed } \\
& Slow & Regular & Maximum \\
\hline Input distance & Short & Good & Excellent & Excellent \\
& Mean & Good & Good & Excellent \\
& Regular & Regular & Good & Good \\
& Maximum & Critical & Regular & Good \\
& Review & Critical & Critical & Critical
\end{tabular}

Notice that if the fuzzy set is symmetric, then the estimated travel time will be the same as the original value. This method is only useful if the Fuzzy set is asymmetric. If it is asymmetric to the right $(c-b>b-a)$ then the estimated travel time is larger than the original time and vice versa. In our case, we set $b$ equal to the input travel time, $a=0.95 \times b$ and $c=1.15 \times b$. In this formulation, the estimated travel time is always an over estimation of the original travel time given by time and distance. In what follows, we consider a more robust formulation of uncertainty using an FIS.

\section{DSM With FIS}

This is the main contribution of this paper, where a Mamdani FIS is used to determine the travel time between the demand point and the ambulance. The basic FIS system (Zadeh, 2015; Grande et al., 2017; Paramo-Carranza et al., 2017; Rubio, 2017) is composed as shown in Figure 3.

Two inputs are considered for the FIS. First is the distance between the demand point and the ambulance, and the second is given by the speed of the ambulance. The output of the FIS is the travel time. The linguistic values are shown in Figures 4-6.

In the fuzzification stage, the data are transformed into linguistic terms (fuzzy sets). The inference system simulates the human reasoning process by making fuzzy inference on the inputs with IF-THEN rules, and the defuzzification transforms the fuzzy set into a crisp value.

Fifteen fuzzy rules are proposed, and the centroid defuzzification method is used to obtain the estimated travel time. Table 1 shows the fuzzy rules used. We have four consequents and their interpretation is:

- Excellent: when the demand point is covered in a radius of $4 \mathrm{~min}$.

- Good: when the demand point is covered in a radius of 7 min.

- Regular: when the demand point is covered in a radius of 15 min.

- Critical: when the demand point is out of coverage.

\section{EXPERIMENTS AND RESULTS}

For this work, randomly generated demand points and ambulance locations are used. We use as reference the study by Lahijanian et al. (2016), a square province is assumed with a dimension of $30 \mathrm{~km} \times 30 \mathrm{~km}$.

TABLE 2 | Comparison results for the coverage of the three methods, showing the mean $(\mu)$ and SD.

\begin{tabular}{|c|c|c|c|c|c|c|c|c|c|}
\hline \multirow{2}{*}{$\begin{array}{l}\text { Number of } \\
\text { ambulances }\end{array}$} & \multicolumn{3}{|c|}{ Coverage $(\mu \pm$ SD) $(40 \mathrm{~km} / \mathrm{h})$} & \multicolumn{3}{|c|}{ Coverage $(\mu \pm S D)(60 \mathrm{~km} / \mathrm{h})$} & \multicolumn{3}{|c|}{ Coverage $(\mu \pm$ SD) $(80 \mathrm{~km} / \mathrm{h})$} \\
\hline & DSM & $\begin{array}{l}\text { DSM } \\
\text { TFS }\end{array}$ & $\begin{array}{l}\text { DSM } \\
\text { FIS }\end{array}$ & DSM & $\begin{array}{l}\text { DSM } \\
\text { TFS }\end{array}$ & $\begin{array}{l}\text { DSM } \\
\text { FIS }\end{array}$ & DSM & $\begin{array}{l}\text { DSM } \\
\text { TFS }\end{array}$ & $\begin{array}{l}\text { DSM } \\
\text { FIS }\end{array}$ \\
\hline 10 & $25.45 \pm 6.54$ & $19.94 \pm 7.28$ & $28.52 \pm 6.74$ & $77.05 \pm 3.92$ & $74.14 \pm 4.04$ & $99.30 \pm 1.03$ & $97.16 \pm 1.70$ & $95.68 \pm 2.09$ & $100 \pm 0.00$ \\
\hline 15 & $56.33 \pm 5.39$ & $52.24 \pm 6.07$ & $57.94 \pm 4.93$ & $91.93 \pm 2.48$ & $89.56 \pm 2.73$ & $99.96 \pm 0.24$ & $99.93 \pm 0.33$ & $99.90 \pm 0.41$ & $100 \pm 0.00$ \\
\hline 20 & $69.74 \pm 4.95$ & $66.82 \pm 5.22$ & $70.74 \pm 4.83$ & $97.95 \pm 1.80$ & $96.82 \pm 2.18$ & $99.96 \pm 0.24$ & $99.94 \pm 0.31$ & $99.91 \pm 0.40$ & $100 \pm 0.00$ \\
\hline 25 & $76.38 \pm 5.15$ & $73.79 \pm 5.46$ & $77.16 \pm 5.06$ & $98.19 \pm 1.79$ & $97.63 \pm 2.16$ & $99.96 \pm 0.24$ & $99.94 \pm 0.31$ & $99.91 \pm 0.40$ & $100 \pm 0.00$ \\
\hline 30 & $80.18 \pm 5.62$ & $77.82 \pm 5.96$ & $80.87 \pm 5.60$ & $98.19 \pm 1.79$ & $97.63 \pm 2.16$ & $99.96 \pm 0.24$ & $99.94 \pm 0.31$ & $99.91 \pm 0.40$ & $100 \pm 0.00$ \\
\hline
\end{tabular}

Bold values are the best values of the experiment. 
A total of $n=100$ demand points are randomly placed using a continuous uniform distribution within the considered area. Then, $m=30$ points, which represents that the possible bases are placed in the following manner. The square is divided into a $3 \times 3$ grid, and it is assumed that more possible ambulance based will be found in the middle of the province (downtown), generating $2 m / 10$ potentials bases in this zone, and $m / 10$ potential bases are

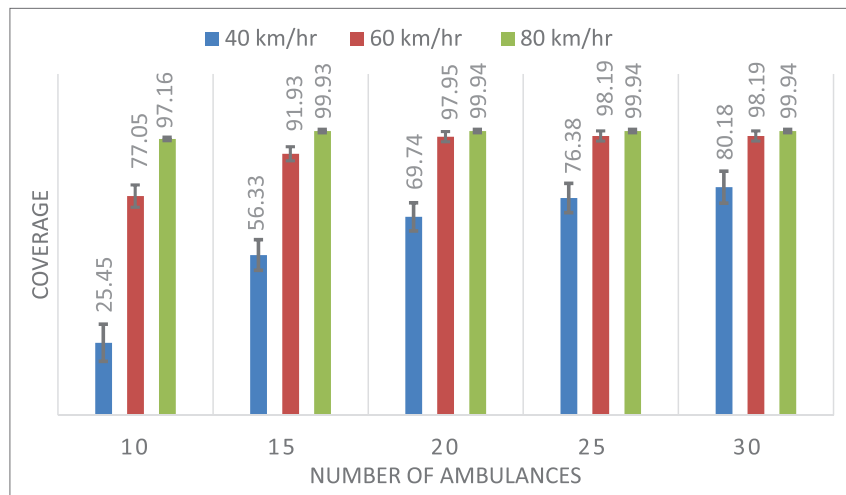

FIGURE 7 | Mean and SD of the coverage using double standard model.

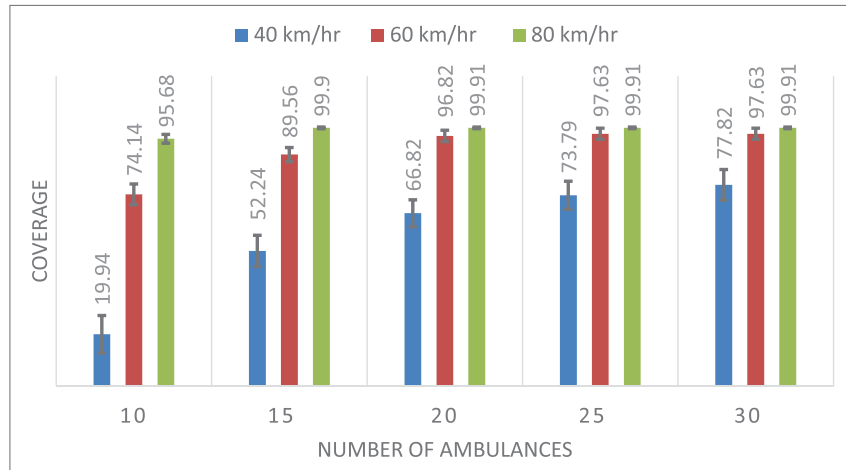

FIGURE 8 | Mean and SD of the coverage using double standard model with triangular fuzzy set.

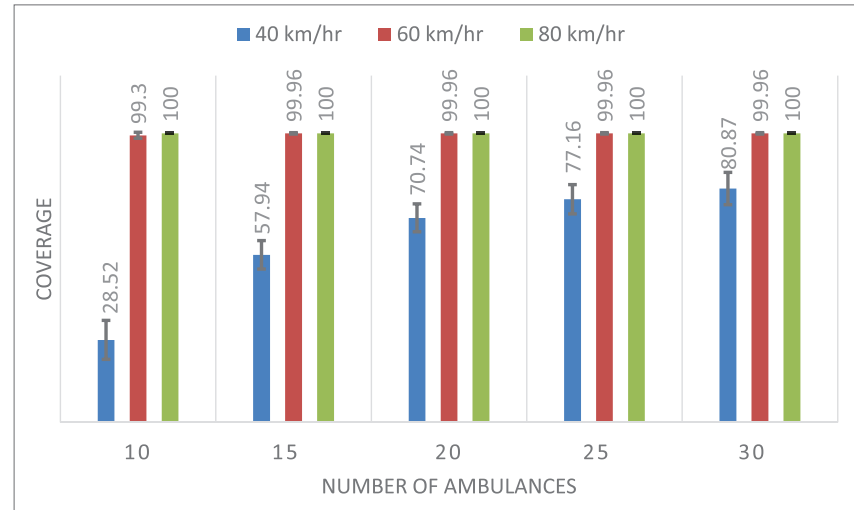

FIGURE 9 | Mean and SD of the coverage using double standard model with fuzzy inference system. generated in each of the eight remaining locations in the grid. Experiments and results are divided into three subsections, which are described below.

\section{Experiment 1: Comparison of the Three Methods Used to Estimate Ambulance Travel Times}

The first group of experiments will try to provide a broad overview of the effect that each method has on the solutions found in the DSM linear program. We will try to get a general characterization by considering different numbers of available ambulances and different top speeds. The following parameters for the DSM are considered:

- $r_{1}: 7$ min (small radius).

- $r_{2}: 15$ min (large radius).

- $p:[10,15,20,25$, and 30], total available ambulances.

- $\alpha$ : 0.65; percentage of total demand which must be covered by an ambulance located within radius $r_{1}$, this parameter is based on the tests carried out in the study by Lahijanian et al. (2016).

To compare the performance of the three methods, we generated 100 different configurations, considering:

- Random distribution of demand points.

- Random distribution of bases.

- Maximum number of ambulances in each base set randomly in the range [1-3].

- Different speeds $(40,60$, and $80 \mathrm{~km} / \mathrm{h})$.

Table 2 shows the mean and SD of the coverage results for each of the three methods considering different speeds. Figures 7-9 show the same comparison showing the mean coverage and SD with error bars, by solving the 100 different problems considering different speeds. Analyzing the results, we can see, in all the cases with different speeds, DSM with FIS method is the best option, the solution is due to the FIS is robust with uncertainty information. These results show that

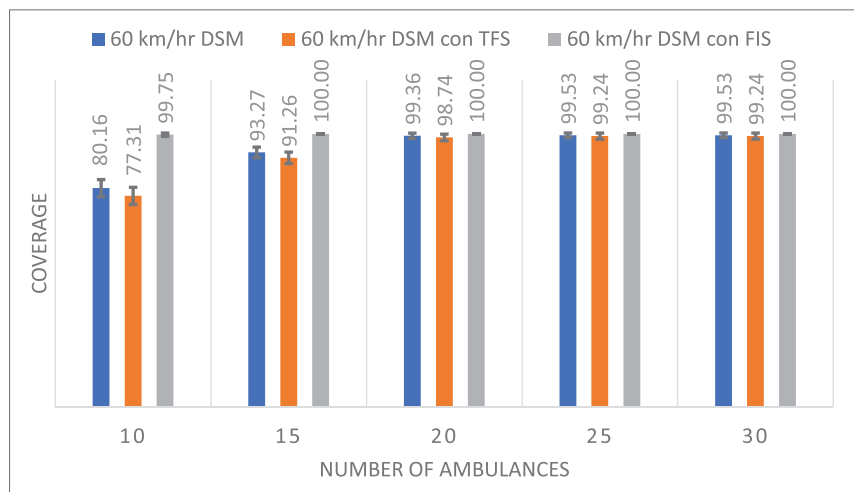

FIGURE 10 | Comparison of coverage (mean and SD) with a speed of $60 \mathrm{~km} / \mathrm{h}$. 
@DSM —DSM with TFS —DSM with FIS

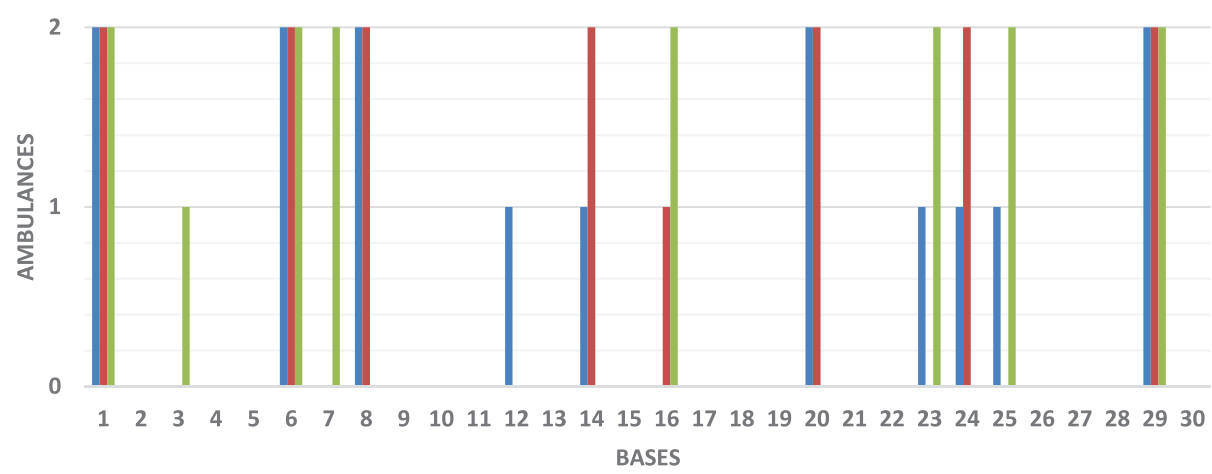

FIGURE 11 | Comparison of number of ambulances located in each base with 15 available ambulances.

the best results are obtained when using the FIS to estimate the travel times. Moreover, these results seem to be quite consistent, independent of the number of available ambulances and the speed of the ambulances.

\section{Experiment 2: Comparison in a Typical Scenario}

In this case, we are interested in analyzing a more typical scenario. We repeat the experiments described in Experiment 1, with the following two modifications. First, the maximum number of ambulances allowed in each base is set to $p_{j}=2$. Second, the speed of the ambulance is set to $60 \mathrm{~km} / \mathrm{h}$. These settings are consistent with those suggested by the RCT.

Figure 10 shows the average results of 100 randomly generated problems and the SD shown with error bars. Results are clear, the DSM with the FIS method achieves the best results. Notice that with 10 ambulances, the coverage is $99.75 \%$ for this approach, while for $15,20,25$, and 30 ambulances the coverage is $100 \%$.

To compare the differences between each method, we take a single case as an example. We consider a total of 15 available ambulances. Figure 11 shows how the number of ambulances (vertical axis) located in each base (horizontal axis). In this case, double coverage with the standard DSM is $93 \%, 91 \%$ for the DSM using a TFS, and $100 \%$ for the DSM using a FIS to account for uncertainty in travel time.

\section{Experiment 3: Analysis of the DSM With FIS}

Let us now provide a more detailed discussion of the result achieved for the DSM with the FIS. Figure 12 shows the problem used for the comparison in Figure 11, where the DSM with FIS achieved $100 \%$ coverage. The triangles represent the 30 bases and the circles indicate the 100 demand points. The distribution of ambulances, as also seen in Figure 11, is: Base 1, Base 6, Base 7, Base 16, Base 23, Base 25, and Base 29 with 2 ambulances and Base 3 with 1 ambulance (a total of 15 ambulances).

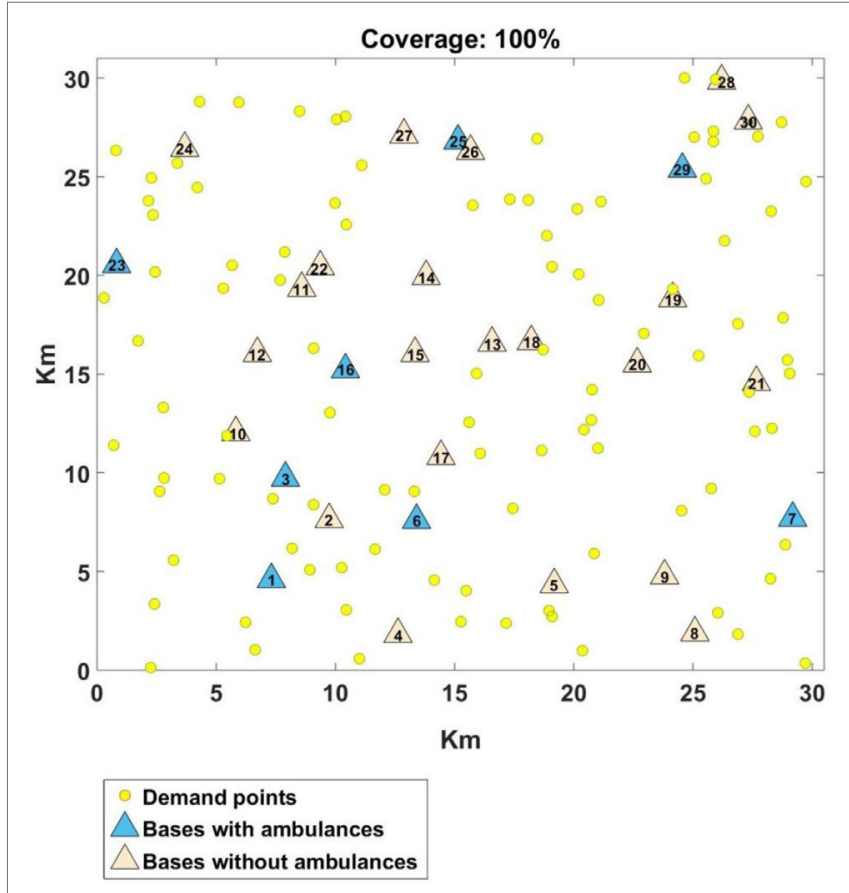

FIGURE 12 | Distribution of ambulances found by the double standard model with fuzzy inference system.

Considering the consequents of the fuzzy rules used by the FIS, Figure 13 shows the number of demand points that are covered by each base in a time that is considered to be excellent, good, regular, or critical, as defined in the Section "DSM with FIS."

Now, considering the small radius in the DSM, we can provide a more detailed analysis of the total coverage achieved. Figure 14 shows the number of demand points covered by each base within the small radius. Notice that all bases cover no less than 10 demand points, with some based covering as much as 30 demand points. Conversely, Figure 15 shows the number of bases that provide coverage to each demand point within the small radius. 

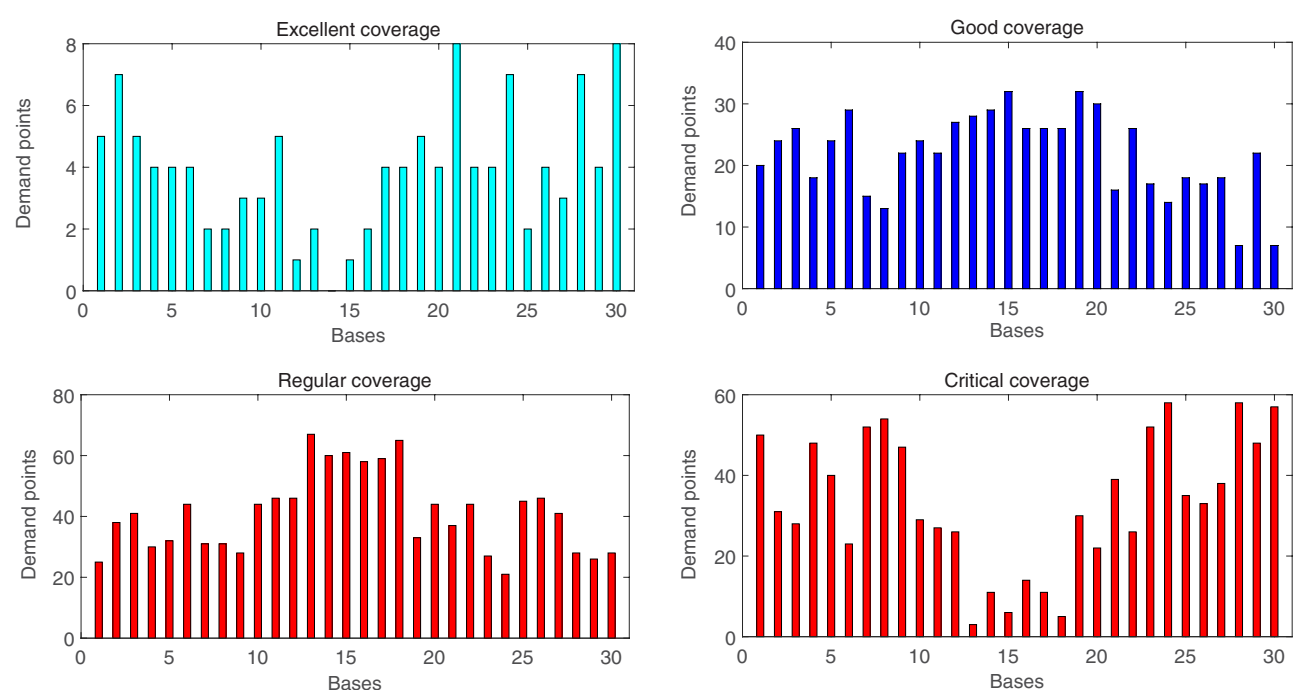

FIGURE 13 | Number of demand points covered by each base, considering different possible fuzzy consequents (excellent, good, regular, and critical).

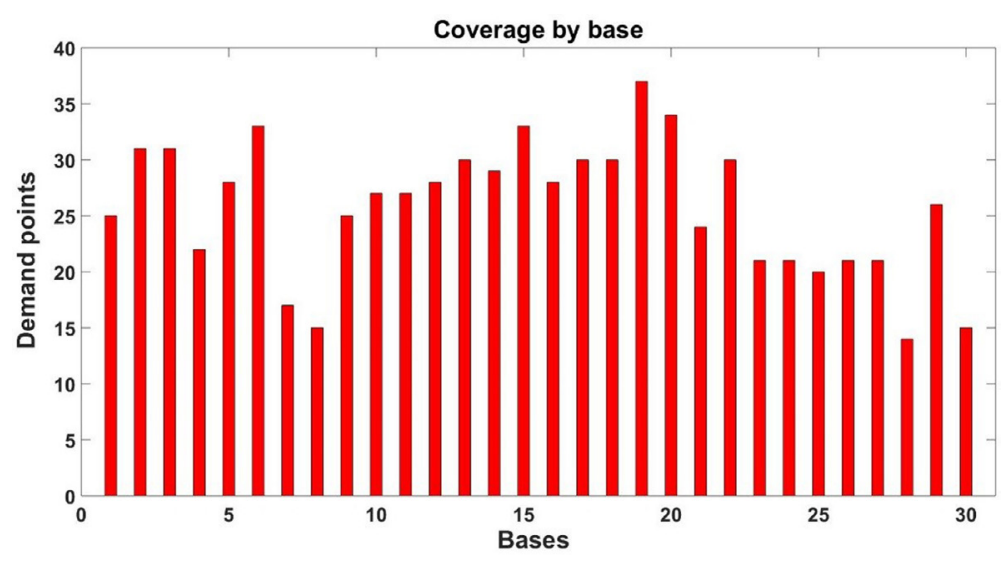

FIGURE 14 | Demand points that cover each base.

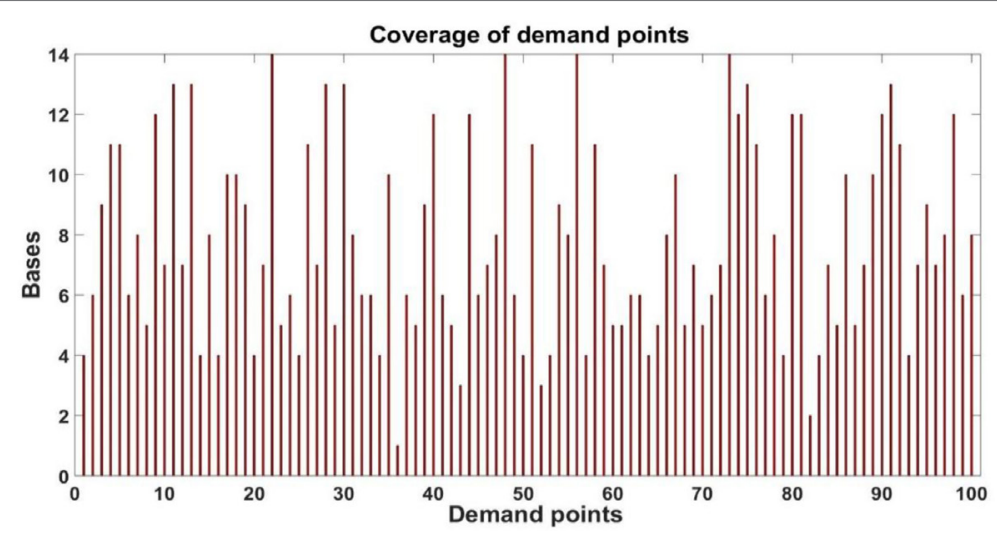

FIGURE 15 | Number of bases covering demand points. 


\section{CONCLUSION AND FUTURE WORK}

In this work, the uncertainty is introduced into in the estimated travel time between a demand point and a candidate base for the ambulance location problem solved with the DSM. Three methods are compared, using no uncertainty, modeling uncertainty with a TFS, and a more complete approach that used an FIS. The coverage obtained with the DSM improves when uncertainty is considered, particularly using the FIS which achieves 100\% coverage in cases where the standard approach does not. These results clearly indicate the importance of considering uncertainty in this real-world domain.

In future work, we will focus on applying the proposed method on real data taken from the EMS of the RCT. The application of the model will improve the location of ambulances and the response time to emergency services will be

\section{REFERENCES}

Braun, O., McCallion, R., and Fazackerley, J. (1990). Characteristics of midsized urban EMS systems. Ann. Emerg. Med. 19, 536-546. doi:10.1016/ S0196-0644(05)82186-9

Church, R., and ReVelle, C. (1974). The maximal covering location problem. Reg. Sci. Assoc. 32, 101-118. doi:10.1111/j.1435-5597.1974.tb00902.x

Cruz Roja de Tijuana. (2012). Estadísticas realizadas por la Cruz Roja Tijuana. Tijuana, BC: Cruz Roja de Tijuana.

Davari, S., Zarandi, M. H. F., and Hemmati, A. (2011). Maximal covering location problem (MCLP) with fuzzy travel times. Exp. Syst. Appl. 38, 14535-14541. doi:10.1016/j.eswa.2011.05.031

Dibene, J. C., Maldonado, Y., Vera, C., de Oliveira, M., Trujillo, L., and Schuetze, O. (2017). Optimizing the location of ambulances in Tijuana, Mexico. Comput. Biol. Med. 80, 107-113. doi:10.1016/j.compbiomed.2016.11.016

Eiben, A. E., and Smith, J. E. (2015). Introduction to Evolutionary Computing. Berlin, Heidelberg: Springer-Verlag.

EMSWorld.com. (2014). EMS Response Time Standards. Available at: http://www. emsworld.com/article/10324786/ems-response-time-standards (Accessed: August 07, 2017).

Gendrau, M., Laporte, G., and Semet, F. (1997). Solving an ambulance location model by Tabu search. Loc. Sci. 5, 75-88. doi:10.1016/S0966-8349(97)00015-6

Grande, A., Hernandez, T., Curtidor, A. V., Paramo, L. A., Tapia, R., Cazares, I. O., et al. (2017). Analysis of fuzzy observability property for a class of TS fuzzy models. IEEE Latin Am. Trans. 15, 595-602. doi:10.1109/ TLA.2017.7896343

INEGI. (2010). Censo de población y vivienda 2010. Available at: www.inegi.org. $\mathrm{mx} /$ est/lista_cubos/consulta.aspx? $\mathrm{p}=$ pob\&c=1 (Accessed: August 07, 2017).

Jones, G. (2002). Genetic and Evolutionary Algorithms. UK: Encyclopedia of Computational Chemistry.

Lahijanian, B., Zarandi, M. H. F., and Farahani, F. V. (2016). "Double coverage ambulance location modeling using fuzzy traveling time," in Fuzzy Information Processing Society (NAFIPS). El Paso, Tx: Annual Conference of the North American.

Laporte, G., Louveaux, F. V., Semet, F., and Thirion, A. (2009). Application of the Double Standard Model for Ambulance Location. in: Innovations in Distribution Logistics. Lecture Notes in Economics and Mathematical Systems, vol 619, eds J. Nunen, M. Speranza, L. Bertazzi, Berlin, Heidelberg: Springer.

Li, X., Zhao, Z., Zhu, X., and Wyatt, T. (2011). Covering models and optimization techniques for emergency response facility location and planning: a review. Math. Methods Oper. Res. 74, 281-310. doi:10.1007/s00186-011-0363-4 timely, a most important problem in a city where resources are scarce.

\section{AUTHOR CONTRIBUTIONS}

NT studied and implemented the algorithms to solve the ambulance location problem and implemented the fuzzy approach to travel time uncertainty. YM and LT were responsible for analyzing the results obtained.

\section{ACKNOWLEDGMENTS}

The authors acknowledge the support of CONACYT, Tecnológico Nacional de México with the project 6350.17-P and CONACYT with the project FC-2015-2/944 Aprendizaje evolutivo a gran escala.

Luke, S. (2014). Essentials of Metaheuristics. Creative Commons. 2nd Ed. Lulu. Available at: https://cs.gmu.edu/ sean/book/metaheuristics/Essentials.pdf

MatLab. (2015). Linear Programming. Available at: https://es.mathworks.com/ discovery/linear-programming.html (Accessed: August 07, 2017).

OMG. (2015). Informe sobre la situación mundial de la seguridad vial. Suiza: OMS.

Paramo-Carranza, L. A., Meda-Campaña, J. A., Rubio, J. J., Tapia Herrera, R., Curtidor Lopez, A. V., Grande Meza, A., et al. (2017). Discrete-time kalman filter for Takagi-Sugeno fuzzy models. Envol. Syst. 8, 211-219. doi:10.1007/ s12530-017-9181-0

Rezaei, M., and Zarandi, M. H. F. (2011). Facility location via fuzzy modeling and simulation. Appl. Soft Comput. 11, 5330-5340. doi:10.1016/j.asoc.2011. 05.026

Rubio, J. J. (2017). USNFIS: uniform stable neuro fuzzy inference system. Neurocomputing 2017, 57-66. doi:10.1016/j.neucom.2016.08.150

Schmid, V. (2012). Solving the dynamic ambulance relocation and dispatching problem using approximate dynamic programming. Eur. J. Oper. Res. 219, 611-621. doi:10.1016/j.ejor.2011.10.043

Shiah, D. M., Hung, C. T., and Chen, S. W. (2009). "Multi-capacities ambulance location model," in Testbeds and Research Infrastructures for the Development of Networks \& Communities and Workshops (IEEE TridentCom), 2009. Washington, DC.

Toregas, C., Swain, R., ReVelle, R., and Bergman, L. (1971). The location of emergency service facilities. Oper. Res. 19, 1363-1373. doi:10.1287/opre.19.6.1363

Zadeh, L. A. (2015). Fuzzy logic, a personal perspective. Fuzzy Sets Syst. 281, 4-20. doi:10.1016/j.fss.2015.05.009

Zarkeshzadeh, M., Zare, H., Heshmati, Z., and Teimouri, M. (2016). A novel hybrid method for improving ambulance dispatching response time through a simulation study. Simulat. Model. Prac. Theory 60, 170-184. doi:10.1016/j. simpat.2015.10.004

Conflict of Interest Statement: The authors declare that the research was conducted in the absence of any commercial or financial relationships that could be construed as a potential conflict of interest.

Copyright (c) 2018 Torres, Trujillo and Maldonado. This is an open-access article distributed under the terms of the Creative Commons Attribution License (CC BY). The use, distribution or reproduction in other forums is permitted, provided the original author(s) and the copyright owner are credited and that the original publication in this journal is cited, in accordance with accepted academic practice. No use, distribution or reproduction is permitted which does not comply with these terms. 